\title{
Safety evaluation of poly(lactic-co-glycolic acid)/ poly(lactic-acid) microspheres through intravitreal injection in rabbits
}

\author{
This article was published in the following Dove Press journal: \\ International Journal of Nanomedicine \\ 24 June 2014 \\ Number of times this article has been viewed
}

\author{
Xianfang Rong' \\ Weien Yuan ${ }^{2}$ \\ Yi Lu' \\ Xiaofen Mo' \\ 'Department of Ophthalmology and \\ Vision Science, Eye and ENT Hospital, \\ Shanghai Medical College, Fudan \\ University, Shanghai, People's Republic \\ of China; ${ }^{2}$ School of Pharmacy, \\ Shanghai Jiao Tong University, \\ Shanghai, People's Republic of China
}

\begin{abstract}
Poly(lactic-co-glycolic acid) (PLGA) and/or poly(lactic-acid) (PLA) microspheres are important drug delivery systems. This study investigated eye biocompatibility and safety of PLGA/PLA microspheres through intravitreal injection in rabbits. Normal New Zealand rabbits were randomly selected and received intravitreal administration of different doses (low, medium, or high) of PLGA/PLA microspheres and erythropoietin-loaded PLGA/PLA microspheres. The animals were clinically examined and sacrificed at 1, 2, 4, 8, and 12 weeks postadministration, and retinal tissues were prepared for analysis. Retinal reactions to the microspheres were evaluated by terminal deoxynucleotidyl transferase-mediated dUTP nick end staining and glial fibrillary acidic protein immunohistochemistry. Retinal structure changes were assessed by hematoxylin and eosin staining and transmission electron microscopy. Finally, retinal function influences were explored by the electroretinography test. Terminal deoxynucleotidyl transferase-mediated dUTP nick end staining revealed no apoptotic cells in the injected retinas; immunohistochemistry did not detect any increased glial fibrillary acidic protein expression. Hematoxylin and eosin staining and transmission electron microscopy revealed no micro- or ultrastructure changes in the retinas at different time points postintravitreal injection. The electroretinography test showed no significant influence of scotopic or photopic amplitudes. The results demonstrated that PLGA/PLA microspheres did not cause retinal histological changes or functional damage and were biocompatible and safe enough for intravitreal injection in rabbits for controlled drug delivery.
\end{abstract}

Keywords: PLGA/PLA microspheres, intravitreal injection, posterior segment diseases, biocompatibility

\section{Introduction}

Drug delivery, especially protein drug delivery to the posterior segment of the eye, has been one of the most challenging endeavors for both ophthalmologists and pharmaceutical scientists. Because of the anatomical structure and physiology of the eye, the blood-ocular barrier is the main reason why it is difficult to deliver drugs to the posterior segment. ${ }^{1}$ Systemic administration and delivery via eye drops and periocular injection make it difficult to achieve an effective therapeutic protein drug concentration. Tear production, nonproductive absorption, and transient residence time also reduce the bioavailability of drugs. ${ }^{2-4}$ Although topical therapy for posterior segment diseases has been proven successful, ${ }^{5}$ and systemic administration of drugs can be used for retinal disorders, ${ }^{6,7}$ the accessibility of ocular tissues is greatly limited, and high systemic doses are needed. This increases drug exposure in nonocular tissues and enhances the risk of systemic side effects. ${ }^{8}$ 
Intravitreal injection is an effective means of delivering therapeutic levels of drugs in a timely manner with minimal systemic side effects. However, most drugs have a limited half-life, and frequent injections are often needed to maintain the vitreous concentrations of drugs in the therapeutic range. Repeated invasive administration may increase ocular complications associated with the procedure, such as vitreous hemorrhage, retinal detachment, cataract, and endophthalmitis, which can significantly influence vision function. ${ }^{9}$

The controlled drug delivery systems that have been developed to overcome these limitations offer an excellent alternative to multiple intravitreal injections. Such systems can provide prolonged therapeutic drug concentrations in ocular tissues while limiting systemic exposure, side effects, and the high peak intraocular drug concentrations associated with pulsed dosing. These systems may also improve patient compliance. ${ }^{10,11}$ The most popular dosing device is the microsphere, which is made up of poly(lactic-co-glycolic acid) (PLGA) and/or poly(lactic acid) (PLA). ${ }^{12}$ This device has the inherent advantages of biodegradability and biocompatibility in nonocular tissues; it degrades into metabolic lactic and glycolic acids in vivo and eventually into water and carbon dioxide, which can be eliminated effectively from the body. ${ }^{13,14}$ Previous studies conducted by the present researchers have demonstrated the neuroprotective effects of the intravitreal protein drug delivery system (erythropoietin [EPO]-dextran PLGA/PLA microspheres) on retinal ganglion cells in rats. ${ }^{15}$ The microspheres were first prepared by a unique system of stabilized aqueous-aqueous "emulsion" to formulate EPOdextran particles. The particles were then encapsulated into PLGA/PLA by the solid-in-oil-in-water (S/O/W) method. ${ }^{16}$ The manufacturing procedure realized the prolonged release of protein and addressed the protein denature or aggregation during the preparing and releasing process. Other studies have also been published on the injection of PLGA microsphere drug delivery systems in retinal neurodegenerative diseases. ${ }^{17-19}$ However, the effects of PLGA and/or PLA microspheres and their biodegraded products in the vitreous cavity on the normal retina and on vision function are not well documented. Therefore, the purpose of this study was to explore eye biocompatibility and the safety of PLGA/PLA microspheres through intravitreal injection in normal New Zealand rabbits by evaluating retinal histological and functional changes.

\section{Materials and methods Materials}

Dextran 64,000-70,000 of average molecular weight and Tween 20 were supplied by Sigma-Aldrich Co. (St Louis, MO, USA). Polyethylene glycol 8000 of average molecular weight (PEG 8000) and EPO were purchased from Shanghai Clonbiotech Co. Ltd (Shanghai, People's Republic of China). PLGA (47 kDa, 5050 DL, inherent viscosity: 0.25-0.43 dL/g) and low-viscosity PLA were obtained from Lakeshore Biopolymers Inc. (Cincinnati, OH, USA). Polyvinyl alcohol (PVA), dichloromethane (DCM), and sodium chloride $(\mathrm{NaCl})$ were obtained from the Chinese Medicine Group Chemical Reagent Corporation (Shanghai, People's Republic of China). Santen Co. Ltd (Osaka, Japan) provided $0.4 \%$ oxybuprocaine, $0.3 \%$ ofloxacin ophthalmic ointment, 1\% tropicamide, and 5\% phenylephrine. An optimal cutting temperature compound was purchased from Ted Pella, Inc. (Tissue-Tek ${ }^{\circledR}$; Redding, CA, USA). An in situ apoptosis detection kit was obtained from Calbiochem of Merck KGaA (Darmstadt, Germany). Mounting media containing 4',6-diamidino-2-phenylindole (DAPI) was purchased from Roche Applied Science (Mannheim, Germany). Bovine serum albumin and goat serum were obtained from Boster Biotechnology Co. Ltd (Wuhan, People's Republic of China). Monoclonal mouse antirabbit primary antibody to glial fibrillary acidic protein (GFAP) and goat antimouse fluorescent secondary antibody were obtained from Molecular Probes ${ }^{\circledR}$ of Thermo Fisher Scientific (Waltham, MA, USA).

\section{Preparation of PLGA/PLA microspheres}

Protein drug-loaded PLGA/PLA microspheres were prepared as previously mentioned. ${ }^{6,15}$ In this study, EPO was used as an example of protein drugs. First, a temperature-induced phase separation method was applied to prepared EPO-dextran particles. A cosolution containing EPO (0.003\% weight/ weight [wt/wt]), dextran (1\% wt/wt), and PEG ( $8 \% \mathrm{wt} / \mathrm{wt})$ was prepared in a $4{ }^{\circ} \mathrm{C}$ water bath and frozen at $-20^{\circ} \mathrm{C}$ for 8-12 hours. The mixture was lyophilized to powder, then resuspended in DCM to dissolve the PEG continuous phase, followed by centrifugation to remove PEG. The pellets were evaporated for 24 hours. Next, EPO-dextran PLGA/ PLA microspheres were prepared using an S/O/W method. The prepared EPO-dextran particles suspended in a DCM solution of PLGA/PLA (PLGA:PLA =4:6, 12.5\% wt/wt) (particles: PLGA/PLA =1:20, wt/wt) were added to $5 \mathrm{~mL}$ of a precooled hydrophilic continuous phase containing $1 \%$ (wt/wt) PVA and $5 \%$ (wt/wt) $\mathrm{NaCl}$ at $4{ }^{\circ} \mathrm{C}$ and stirred at $2,000 \mathrm{rpm}$ for 30 seconds using a magnetic composite. Once microspheres were formed, the sample was immediately transferred into $1 \mathrm{~L}$ of $10 \%$ (wt $/ \mathrm{wt}$ ) $\mathrm{NaCl}$ solution at $0^{\circ} \mathrm{C}$ under gentle stirring (100 rpm) using an electromotive stirrer (Xinhang JJ-1; Jintan Xinhang Co. Ltd, Jintan, People's Republic of China) to extract the organic solvent and harden the embryonic microspheres. This microsphere aging process 
lasted 4 hours. The hardened microspheres were rinsed using distilled water to remove PVA and $\mathrm{NaCl}$ and lyophilized again to remove water and solvent residues. Blank PLGA/ PLA microspheres were prepared in the same manner without EPO dextran particles added.

\section{Animal experiments}

Normal New Zealand rabbits were used to evaluate the biocompatibility and safety of PLGA/PLA microspheres in eyes. All experimental protocols and animal care complied with the Association for Research in Vision and Ophthalmology Statement for the Use of Animals in Ophthalmic and Vision Research and the guidelines of the Animal Care and Use Committee of Fudan University (Shanghai, People's Republic of China). Prior to the intravitreal injection, animals were anesthetized with a mixture of ketamine $(40 \mathrm{mg} / \mathrm{kg}$ body weight) and xylazine ( $4 \mathrm{mg} / \mathrm{kg}$ body weight) by an intramuscular injection. Topical $0.4 \%$ oxybuprocaine eye drops were also administered. Then, PLGA/PLA microspheres were suspended in $0.01 \mathrm{M}$ phosphate-buffered saline (PBS). The animals were divided into six groups. Each received a single intravitreal injection of 1) low group: PLGA/PLA microspheres $2.5 \mathrm{mg} / 0.1 \mathrm{~mL}$; 2) medium group: PLGA/ PLA microspheres $5 \mathrm{mg} / 0.1 \mathrm{~mL}$; 3 ) high group: PLGA/PLA microspheres $10 \mathrm{mg} / 0.1 \mathrm{~mL}$; 4) EPO group: EPO-dextran PLGA/PLA microspheres $5 \mathrm{mg} / 0.1 \mathrm{~mL}$; 5) PBS group: 0.01 M PBS $0.1 \mathrm{~mL}$; and 6) normal group: intact eyes received no intravitreal injection. After the procedure, $0.3 \%$ ofloxacin ophthalmic ointment was applied to the ocular surface to prevent infection and corneal desiccation. Slit lamp and indirect funduscopic examinations were performed on each eye immediately following the injection, every day within the first week of the injection, and once a week after 1 week postinjection.

\section{Terminal deoxynucleotidyl transferase- mediated dUTP nick end labeling}

Eyes ( $n=4$ for each group at each time point) enucleated at $1,2,4,8$, and 12 weeks following injection were fixed in $4 \%$ paraformaldehyde for 2 hours at room temperature. After being dehydrated in graded sucrose solution overnight at $4^{\circ} \mathrm{C}$, the eyecups with the removal of the anterior segments were embedded in optimal cutting temperature compound at $-20^{\circ} \mathrm{C}$. Cryostat sections of $10 \mu \mathrm{m}$ thickness were obtained. To detect whether there was retinal ganglion cell death caused by the microspheres and their degraded products, terminal deoxynucleotidyl transferase-mediated dUTP nick end (TUNEL) labeling was performed by an in situ apoptosis detection kit (Calbiochem ${ }^{\circledR}$, Merk KGaA,
Darmstadt, Germany) according to the manufacturer's protocol. Mounting media containing DAPI was used to image the nuclei. All preparations were observed under a fluorescent microscope (Leica DM4000B; Leica Microsystems, Wetzlar, Germany), and six slides for each eye were prepared.

\section{GFAP immunohistochemistry}

Cryostat sections were obtained as described previously, and six slides were randomly chosen from each eye $(n=4$ for each group at each time point) at 1, 2, 4, 8, and 12 weeks after the injection, respectively. GFAP immunohistochemistry staining was performed. For antigen retrieval, sections were first put in $0.01 \%$ Tween 20 at room temperature for 5 minutes. They were then incubated in a mixture of $5 \%$ bovine serum albumin and $1 \%$ goat serum for 1 hour to reduce nonspecific binding. Then, monoclonal mouse antirabbit primary antibody to GFAP (1:200) was used at room temperature for 24 hours. After being washed three times in 0.01 M PBS, goat antimouse fluorescent secondary antibody (1:500) was applied for 1 hour. Followed by three washes in 0.01 M PBS, the sections were stained with $1 \mu \mathrm{g} / \mathrm{mL}$ DAPI in methanol for 5 minutes to label cell nuclei. The sections were then observed under a fluorescence microscope.

\section{Hematoxylin and eosin staining}

Eyes ( $n=4$ for each group at each time point) were enucleated at 1, 2, 4, 8, and 12 weeks after intravitreal injection and fixed for 24 hours at $4{ }^{\circ} \mathrm{C}$. Following fixation, the anterior segments were removed. The posterior eyecups were dehydrated through a graded alcohol series, cleared in xylene, and embedded in paraffin. Serial slides of $3 \mu \mathrm{m}$ were cut, and six slides for each eye were randomly chosen and stained with hematoxylin and eosin (HE). A microscope (Leica DM4000B; Leica Microsystems) was used for general observation of microstructure changes of the retinas. The sections were photographed at approximately two to three disc diameters from the optic nerve.

\section{Transmission electron microscopy}

Animals ( $\mathrm{n}=4$ for each group at each time point) were euthanized at different times following intravitreal administration $(1,2$, and 4 weeks postinjection). Each eye was rapidly enucleated and fixed overnight in a solution of $2.5 \%$ glutaraldehyde and $2.5 \%$ paraformaldehyde after the removal of the cornea, lens, and vitreous body. The samples were osmicated and embedded in Epon (Electron Microscopy Sciences, Hatfield, PA, USA) according to standard procedures. Six ultrathin sections were randomly chosen from each eye and stained 
with uranyl acetate and lead citrate and then examined by a Philips CM120 transmission electron microscope (TEM), (Philips Electronics Ltd., Amsterdam, the Netherlands). The eyes were analyzed by masked examiners to avoid bias, and cellular ultrastructure injury on TEM was evaluated.

\section{Electroretinography}

Animals used for electroretinography (ERG) received intravitreal injection in the right eye, whereas the contralateral eyes remained untouched, in order to serve as controls. The ERG was assessed in the low, medium, high, and EPO groups ( $n=4$ for each group at each time point) immediately after the right eye injection and at 1, 2, 4, 8, and 12 weeks after the injection. Rabbits were initially dark-adapted overnight for scotopic response and 10-minute light-adapted before photopic response in both eyes. Pupils were dilated with $1 \%$ tropicamide and 5\% phenylephrine, and the animals were anesthetized by intramuscular injection before the recording of ERG. Two gold cup electrodes coated with $2 \%$ methylcellulose were placed over both corneas, and a needle electrode was inserted into the back. Scotopic and photopic ERG responses were recorded, and the results of the left intact eye served as baseline. Visual stimuli were generated by $5 \mathrm{~ms}$ duration flashes of a white light. The responses to a light flash $\left(0.01 \mathrm{~cd} \cdot \mathrm{s} / \mathrm{m}^{2}\right.$ and $1.0 \mathrm{~cd} \cdot \mathrm{s} / \mathrm{m}^{2}$ in scotopic and photopic ERG, respectively) from a photic stimulator were amplified, filtered, and averaged. All examinations were carried out at room temperature and with no evident tachypnea.

\section{ERG analysis}

The scotopic and photopic A-wave amplitude was measured as the difference in amplitude between the recording at onset and the trough of the negative deflection. The B-wave amplitude was measured as the difference in amplitude between the trough of the A-wave to the peak of the B-wave. Differences in the ERG amplitudes between the injected eyes and the intact eyes in all recorded animals were determined by Student's $t$-tests when the variance of the data was homogeneously distributed, or by the Wilcoxon rank sum test when heterogeneously distributed. The data were expressed as the
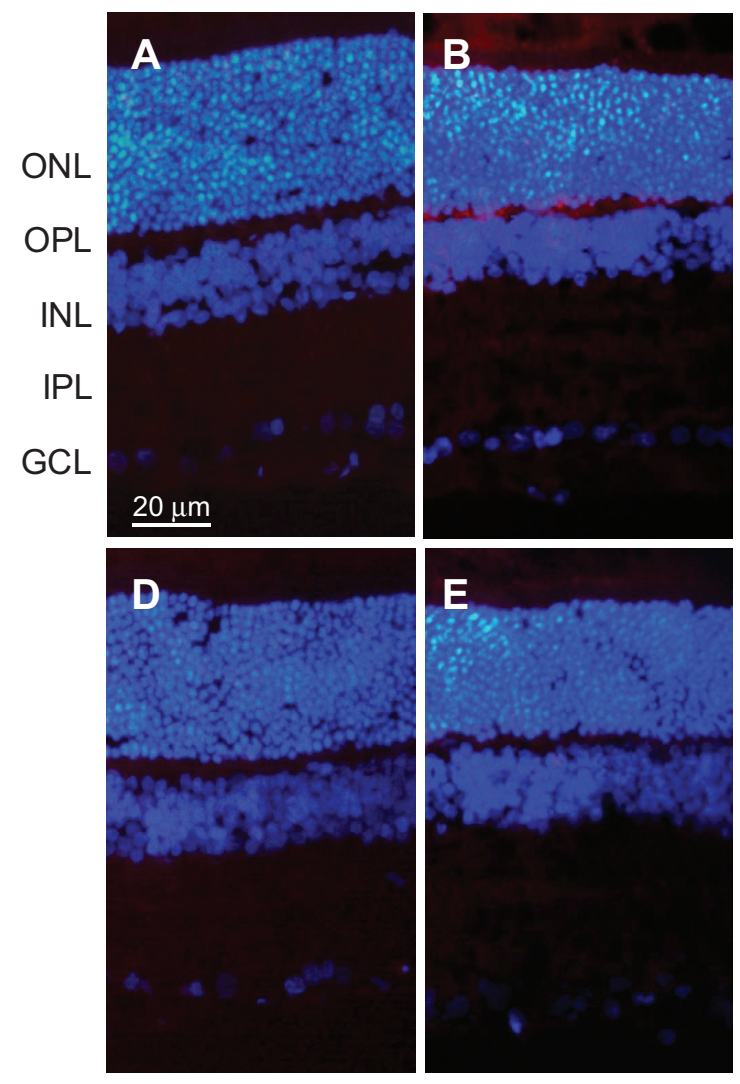
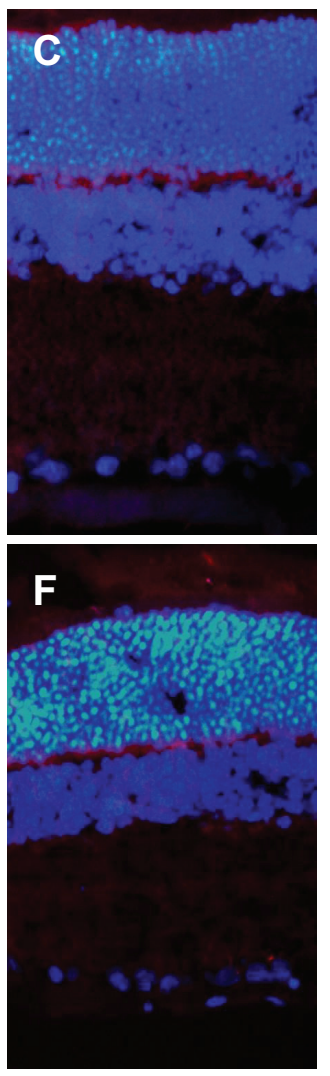

Figure I TUNEL staining for apoptotic cells in retinas at 2 weeks after intravitreal injection.

Notes: (A) Low group: intravitreal injection of $2.5 \mathrm{mg} / 0.1 \mathrm{~mL}$ PLGA/PLA microspheres; (B) medium group: intravitreal injection of $5 \mathrm{mg} / 0.1 \mathrm{~mL}$ PLGA/PLA microspheres; (C) high group: intravitreal injection of $10 \mathrm{mg} / 0.1 \mathrm{~mL}$ PLGA/PLA microspheres; (D) EPO group: intravitreal injection of $5 \mathrm{mg} / 0$. I mL EPO-dextran PLGA/PLA microspheres; (E) PBS group: intravitreal injection of $0.1 \mathrm{~mL} 0.01 \mathrm{M}$ PBS; (F) normal group: normal retinas received no intravitreal injection. No apoptotic-positive cells were found in any of the retinas.

Abbreviations: EPO, erythropoietin; GCL, ganglion cell layer; INL, inner nuclear layer; IPL, inner plexiform layer; ONL, outer nuclear layer; OPL, outer plexiform layer; PBS, phosphate-buffered saline; PLGA/PLA, poly(lactic-co-glycolic acid)/poly(lactic-acid); TUNEL, terminal deoxynucleotidyl transferase-mediated dUTP nick end labeling. 
means \pm standard deviation, and $P<0.05$ was considered statistically significant.

\section{Results}

\section{Clinical observation}

No clinical evidence of ocular changes in the groups of rabbits was noted after intravitreal injection or during the following period, although the microspheres topically remained in the lower part of the vitreous of the injected eyes. These microspheres gradually degraded and disappeared at 11 weeks postinjection. Corneas kept clear, and anterior and posterior media as well as retinas were free of any inflammatory response.

\section{TUNEL labeling}

TUNEL labeling was used to detect cells undergoing apoptotic death induced by the PLGA/PLA and EPO-dextran
PLGA/PLA microspheres or by their degraded products in the vitreous. DAPI was employed to stain cell nuclei in the retinas. The results showed that no TUNEL-positive cells were detected in the DAPI-positive cells in layers of retinas (Figure 1). No deoxyribonucleic acid fragmented nuclei were found in the retinas of all groups at 1, 2, 4, 8, and 12 weeks following intravitreal injection.

\section{GFAP immunohistochemistry}

GFAP expression was mainly localized to the vicinity of the inner limiting membrane and nerve fiber layer in all retinas of intravitreally injected groups, as it was expressed in intact normal retinas. Representative results in Figure 2 show the GFAP expression in retinas at 2 weeks after intravitreal administration. The injection and the microspheres did not cause elevated expression of GFAP, which is a marker of retinal injury. ${ }^{20}$
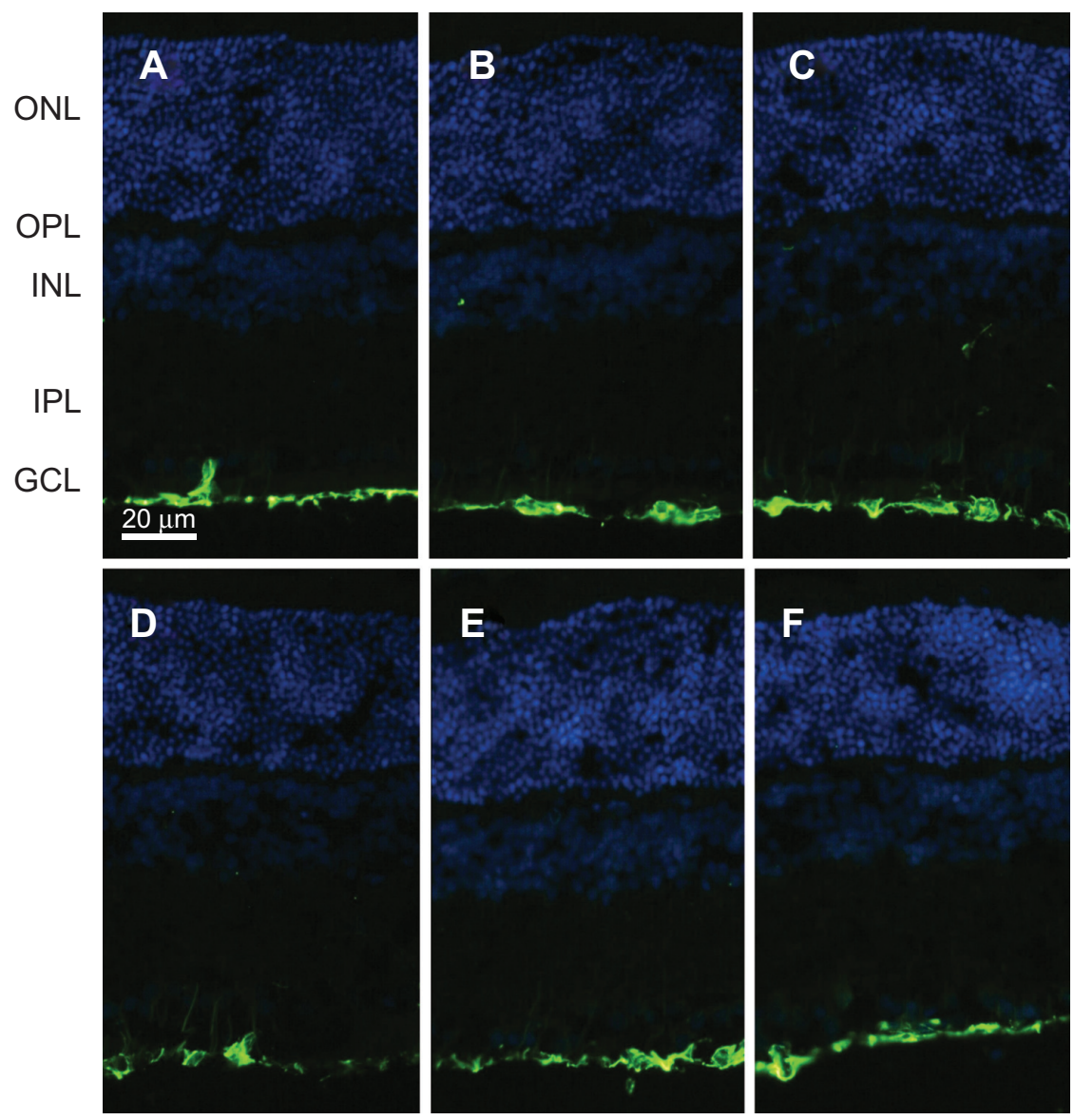

Figure 2 Immunohistochemistry staining of GFAP expressed in retinas at 2 weeks after intravitreal injection.

Notes: (A) Low group: intravitreal injection of $2.5 \mathrm{mg} / 0.1 \mathrm{~mL}$ PLGA/PLA microspheres; (B) medium group: intravitreal injection of $5 \mathrm{mg} / 0.1 \mathrm{~mL}$ PLGA/PLA microspheres; (C) high group: intravitreal injection of $10 \mathrm{mg} / 0.1 \mathrm{~mL}$ PLGA/PLA microspheres; (D) EPO group: intravitreal injection of $5 \mathrm{mg} / 0.1 \mathrm{~mL}$ EPO-dextran PLGA/PLA microspheres; (E) PBS group: intravitreal injection of $0.1 \mathrm{~mL} 0.0$ I M PBS; (F) normal group: normal retinas received no intravitreal injection. GFAP expression was mainly localized in the inner limiting membrane in all groups without any differences between groups.

Abbreviations: EPO, erythropoietin; GCL, ganglion cell layer; GFAP, glial fibrillary acidic protein; INL, inner nuclear layer; IPL, inner plexiform layer; ONL, outer nuclear layer; OPL, outer plexiform layer; PBS, phosphate-buffered saline; PLGA/PLA, poly(lactic-co-glycolic acid)/poly(lactic-acid). 


\section{HE staining}

The microstructure changes in the retinas were examined by HE staining at $1,2,4,8$, and 12 weeks after intravitreal injection. Neither retinal detachment nor inflammatory reactions occurred after the injection of PLGA/PLA or EPOdextran PLGA/PLA microspheres as well as $0.01 \mathrm{M}$ PBS at the examined time points, as represented in Figure 3 at 2 weeks postinjection. The results indicate that the injected microspheres did not trigger an immune reaction in the retinal tissue or any disorganized retinal structure when compared with the normal group.

\section{Transmission electron microscopy}

Ultrastructure changes in retinal tissues were observed by TEM at 1, 2, and 4 weeks postintravitreal injection. No obvious retinal ultrastructure abnormality was noticed between the intravitreally injected groups and the normal retinas at any time points. No evidence of cell toxicity was reflected by the retinal neurons ultrastructure under TEM. The appearance of mitochondria in photoreceptor inner segments and discs in outer segments in injected groups was intact compared with the normal retinas (Figure 4). No mitochondrial swelling, rupture, disc swelling, or disorganization were found.
In retinal ganglion cells, no nuclear pyknosis or karyolysis was detected, except for a small amount of steatosis in cytoplasm, which is often found in intact normal ganglion cells (Figure 5).

\section{Electroretinogram}

An ERG test was applied to evaluate the retinal function influence caused by different doses of PLGA/PLA and EPO-dextran PLGA/PLA microspheres. In the results, no significant decrease in ERG amplitudes (A- and B-waves) was detected in the intravitreally injected eyes compared with the intact eyes at 1, 2, 4, 8, and 12 weeks postinjection in any groups. The ERGs were normal in all eyes with little or no change in A- and B-waves. Representative ERGs are shown in Figures 6, 7, and 8. Figure 8 presents the comparison results of the scotopic and photopic B-wave amplitudes between the injected eyes and the intact eyes at 2 weeks postintravitreal injection, with $P>0.05$.

\section{Discussion}

This study demonstrated the biocompatibility and safety of intravitreally delivered biodegradable PLGA/PLA and EPO-dextran PLGA/PLA microspheres in normal rabbits.

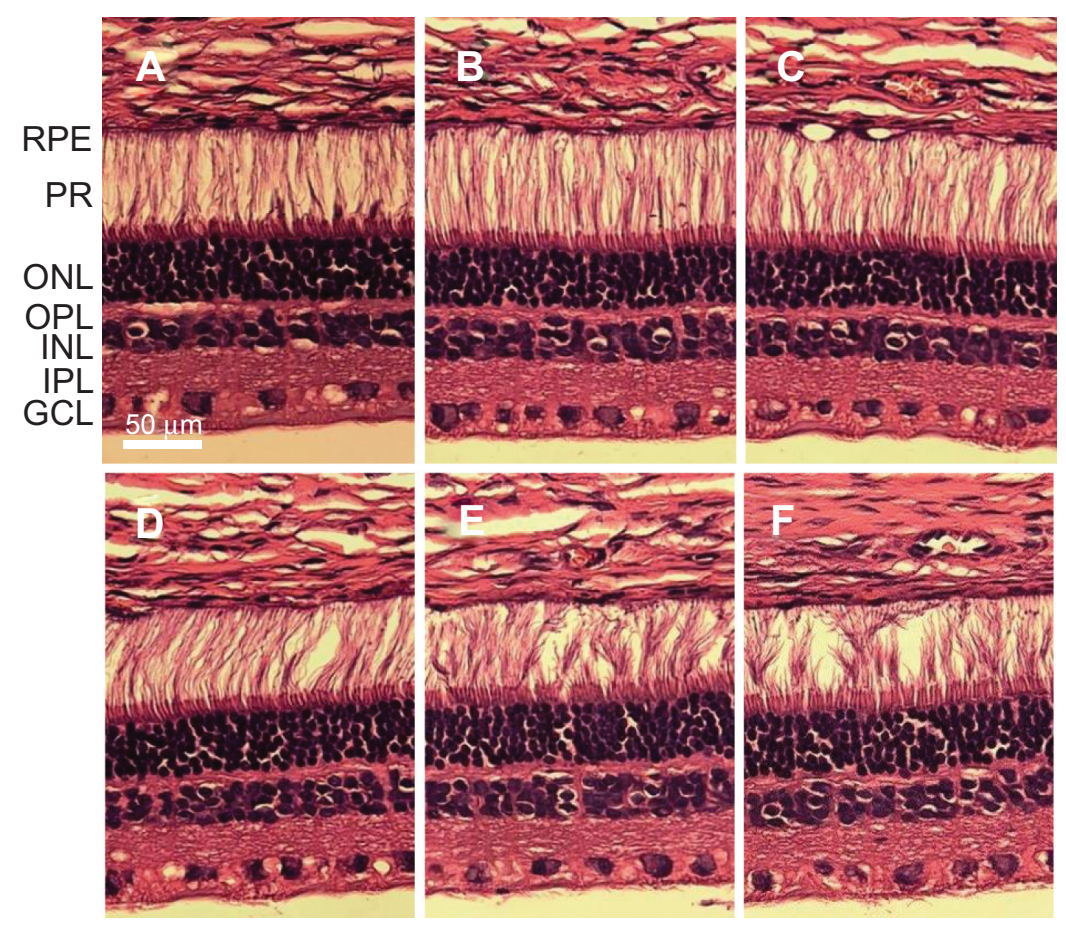

Figure $3 \mathrm{HE}$ staining of structure changes in retinas at 2 weeks after intravitreal injection.

Notes: (A) Low group: intravitreal injection of $2.5 \mathrm{mg} / 0.1 \mathrm{~mL}$ PLGA/PLA microspheres; (B) medium group: intravitreal injection of $5 \mathrm{mg} / 0.1 \mathrm{~mL}$ PLGA/PLA microspheres; (C) high group: intravitreal injection of $10 \mathrm{mg} / 0.1 \mathrm{~mL}$ PLGA/PLA microspheres; (D) EPO group: intravitreal injection of $5 \mathrm{mg} / 0.1 \mathrm{~mL}$ EPO-dextran PLGA/PLA microspheres; (E) PBS group: intravitreal injection of $0.1 \mathrm{~mL} 0.0$ I M PBS; (F) normal group: normal retinas received no intravitreal injection. No obvious structure changes were detected in the retina in the injected groups compared with the intact eyes.

Abbreviations: EPO, erythropoietin; GCL, ganglion cell layer; HE, hematoxylin and eosin; INL, inner nuclear layer; IPL, inner plexiform layer; ONL, outer nuclear layer; OPL, outer plexiform layer; PBS, phosphate-buffered saline; PLGA/PLA, poly(lactic-co-glycolic acid)/poly(lactic-acid); PR, photoreceptor; RPE, retinal pigment epithelium. 

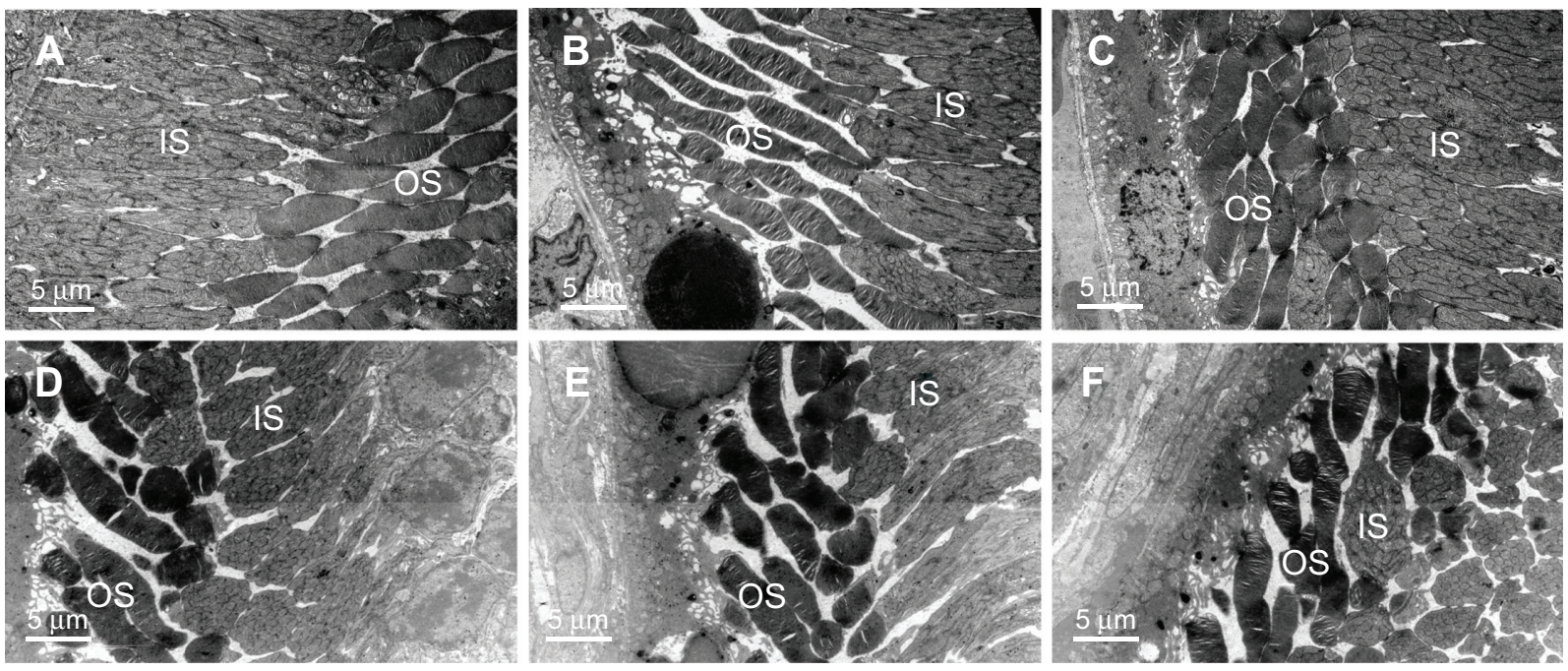

Figure 4 TEM scanning for ultrastructure changes of outer and inner segment of photoreceptors in retinas at 2 weeks after intravitreal injection.

Notes: (A) Low group: intravitreal injection of $2.5 \mathrm{mg} / 0.1 \mathrm{~mL}$ PLGA/PLA microspheres; (B) medium group: intravitreal injection of $5 \mathrm{mg} / 0.1 \mathrm{~mL}$ PLGA/PLA microspheres; (C) high group: intravitreal injection of $10 \mathrm{mg} / 0.1 \mathrm{~mL}$ PLGA/PLA microspheres; (D) EPO group: intravitreal injection of $5 \mathrm{mg} / 0.1 \mathrm{~mL}$ EPO-dextran PLGA/PLA microspheres; (E) PBS group: intravitreal injection of $0.1 \mathrm{~mL} 0.01 \mathrm{M}$ PBS; (F) normal group: normal retinas received no intravitreal injection. No significant morphologic changes were detected in the outer segment disc and inner segment of mitochondria in the groups.

Abbreviations: EPO, erythropoietin; IS, photoreceptor inner segment; OS, photoreceptor outer segment; PBS, phosphate-buffered saline; PLGA/PLA, poly(lactic-coglycolic acid)/poly(lactic-acid); TEM, transmission electron microscope.

The protein-loaded microspheres were manufactured by first loading the drug into dextran particles, then encapsulating the particles in PLGA/PLA microspheres using the S/O/W method. This method realized the prolonged release of proteins and protected the proteins from denaturing or aggregation, as reported previously. ${ }^{16}$ The microspheres possessed a spherical shape, smooth surface, and diameter of $40-100 \mu \mathrm{m}$, with an in vitro release profile of more than 60 days, as analyzed in the previous studies. ${ }^{6,15}$

PLGA- and/or PLA-sustained drug release systems applied in the eye have shown prolonged release of drugs $\mathrm{s}^{21,22}$ and have facilitated effective treatments for chronic ocular disorders such as proliferative vitreoretinopathy, retinal diseases, glaucoma, and neovascularization. . $^{18,19,23,24}$
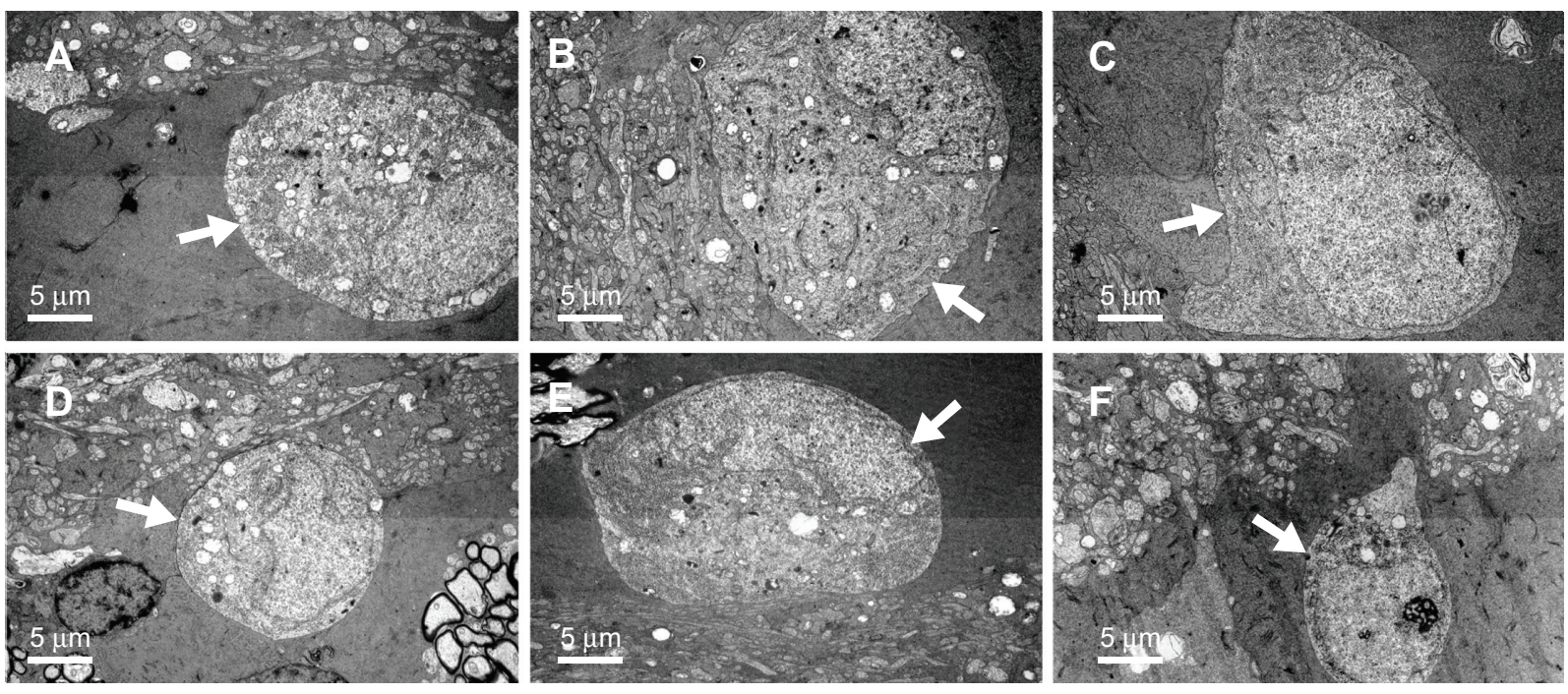

Figure 5 TEM scanning for ultrastructure changes of retinal ganglion cells at 2 weeks after intravitreal injection. Retinal ganglion cells are identified by arrows.

Notes: (A) Low group: intravitreal injection of $2.5 \mathrm{mg} / 0.1 \mathrm{~mL}$ PLGA/PLA microspheres; (B) medium group: intravitreal injection of $5 \mathrm{mg} / 0.1 \mathrm{~mL}$ PLGA/PLA microspheres; (C) high group: intravitreal injection of $10 \mathrm{mg} / 0.1 \mathrm{~mL}$ PLGA/PLA microspheres; (D) EPO group: intravitreal injection of $5 \mathrm{mg} / 0.1 \mathrm{~mL}$ EPO-dextran PLGA/PLA microspheres; (E) PBS group: intravitreal injection of $0.1 \mathrm{~mL} 0.0$ I M PBS; (F) normal group: normal retinas received no intravitreal injection. No distinct morphologic changes were detected in retinal ganglion cells in the injected eyes compared with normal retinas.

Abbreviations: EPO, erythropoietin; PBS, phosphate-buffered saline; PLGA/PLA, poly(lactic-co-glycolic acid)/poly(lactic-acid); TEM, transmission electron microscope. 

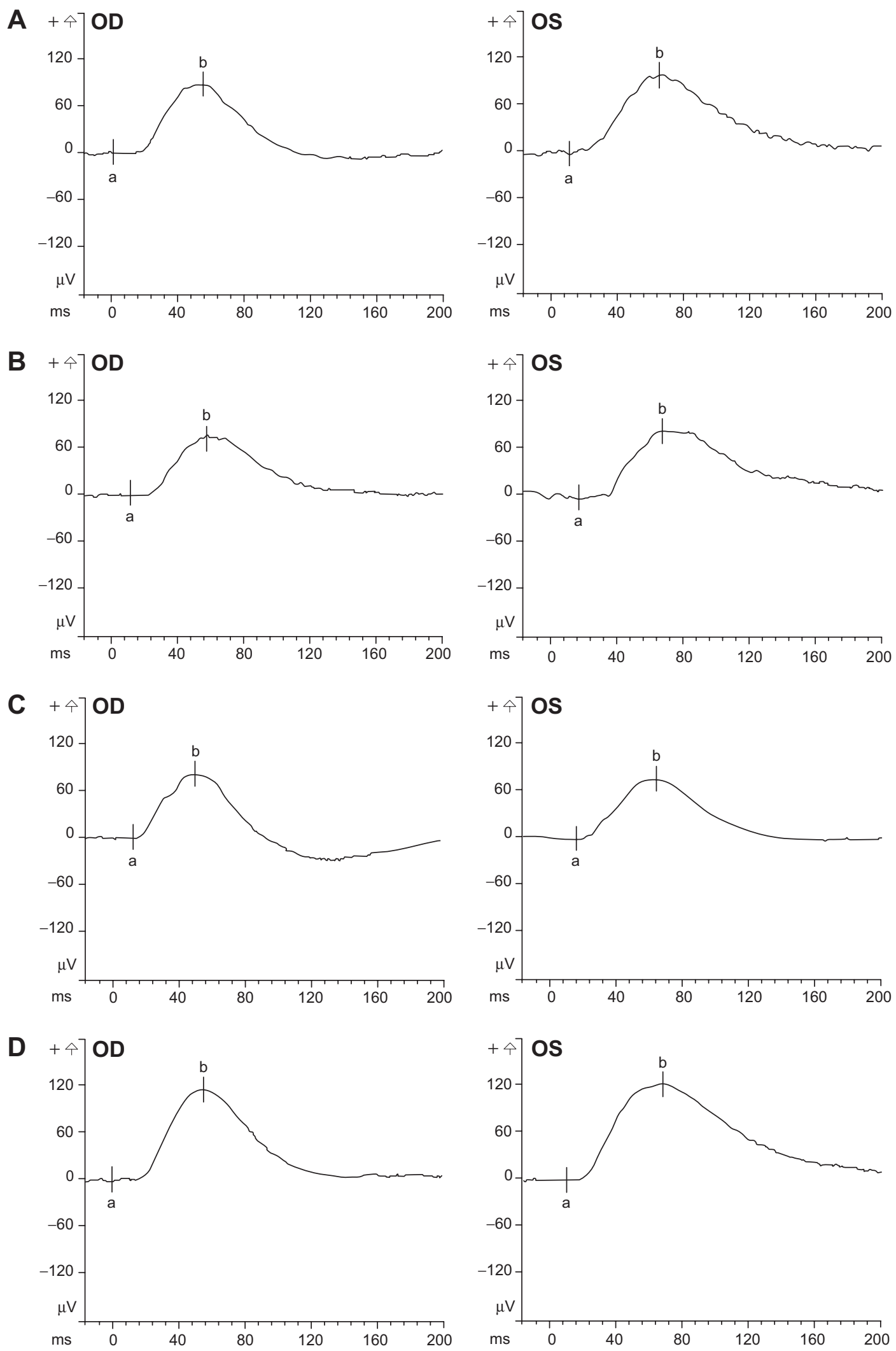

Figure 6 Scotopic ERG of rabbits at 2 weeks after intravitreal injection.

Notes: (A) Low group: intravitreal injection of $2.5 \mathrm{mg} / 0.1 \mathrm{~mL}$ PLGA/PLA microspheres; (B) medium group: intravitreal injection of $5 \mathrm{mg} / 0.1 \mathrm{~mL}$ PLGA/PLA microspheres; (C) high group: intravitreal injection of $10 \mathrm{mg} / 0.1 \mathrm{~mL}$ PLGA/PLA microspheres; (D) EPO group: intravitreal injection of $5 \mathrm{mg} / 0.1 \mathrm{~mL}$ EPO-dextran PLGA/PLA microspheres. No significant scotopic amplitudes were influenced by the injected microspheres compared with the intact eyes.

Abbreviations: a, A-wave of the ERG; b, B-wave of the ERG; EPO, erythropoietin; ERG, electroretinography; OD, the injected right eye; OS, the intact left eye; PLGA/PLA, poly(lactic-co-glycolic acid)/poly(lactic-acid). 

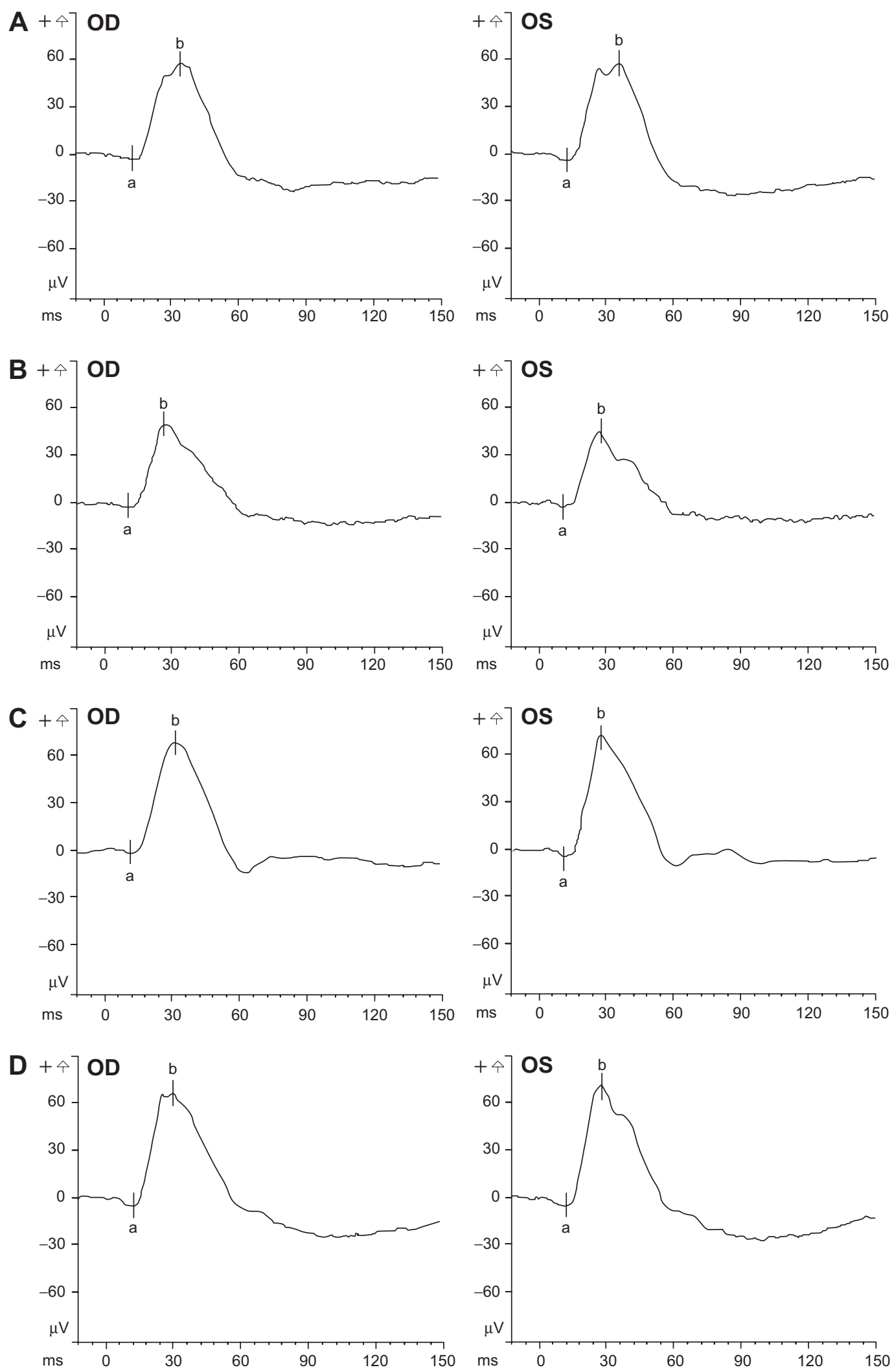

Figure 7 Photopic ERG of rabbits at 2 weeks after intravitreal injection.

Notes: (A) Low group: intravitreal injection of $2.5 \mathrm{mg} / 0.1 \mathrm{~mL}$ PLGA/PLA microspheres; (B) medium group: intravitreal injection of $5 \mathrm{mg} / 0.1 \mathrm{~mL}$ PLGA/PLA microspheres; (C) high group: intravitreal injection of $10 \mathrm{mg} / 0.1 \mathrm{~mL}$ PLGA/PLA microspheres; (D) EPO group: intravitreal injection of $5 \mathrm{mg} / 0.1 \mathrm{~mL}$ EPO-dextran PLGA/PLA microspheres. No significant photopic amplitudes were influenced by the injected microspheres compared with the intact eyes.

Abbreviations: a, A-wave of the ERG; b, B-wave of the ERG; EPO, erythropoietin; ERG, electroretinography; OD, the injected right eye; OS, the intact left eye; PLGA/PLA, poly(lactic-co-glycolic acid)/poly(lactic-acid). 

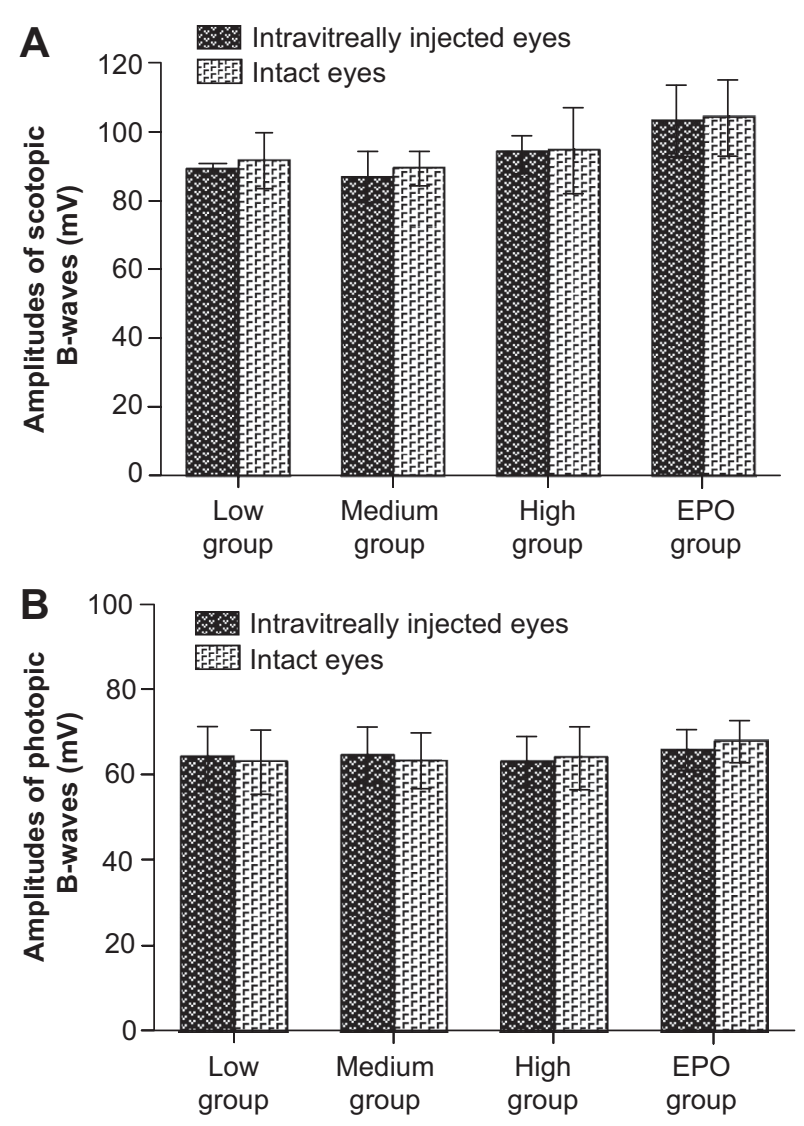

Figure 8 Scotopic (A) and photopic (B) ERG of rabbits at 2 weeks after intravitreal injection. Low group: intravitreal injection of $2.5 \mathrm{mg} / 0.1 \mathrm{~mL}$ PLGA/PLA microspheres; medium group: intravitreal injection of $5 \mathrm{mg} / 0.1 \mathrm{~mL}$ PLGA/PLA microspheres; high group: intravitreal injection of $10 \mathrm{mg} / 0.1 \mathrm{~mL}$ PLGA/PLA microspheres; EPO group: intravitreal injection of $5 \mathrm{mg} / 0.1 \mathrm{~mL}$ EPO-dextran PLGA/PLA microspheres. No statistically significant differences were observed between the intravitreally injected eyes and the intact eyes in all groups.

Abbreviations: EPO, erythropoietin; ERG, electroretinography; PLGA/PLA, poly (DL-lactic-co-glycolic acid)/poly(DL-lactic).

The present researchers' previous studies have revealed that a single intravitreal injection of EPO-dextran PLGA/PLA microspheres could significantly protect retinas damaged from optic nerve crush in rats. ${ }^{15}$ However, whether intravitreally injected PLGA/PLA microspheres and their degraded products could cause inflammatory reactions and damage to retinas and vision function have not been systemically investigated.

In this study, possible ocular reactions after intravitreal injection were observed through clinical ophthalmoscopic examination and retinal cell responses. The clinical results showed that anterior and posterior media of the eyes kept clear, although the microspheres remained topically in the lower part of the vitreous; these microspheres gradually degraded and disappeared at 11 weeks postinjection. No cataract, retinal detachment, or inflammatory vitreous opacity was induced. Meanwhile, in the retinal sections, no apoptotic cells or elevated GFAP expression were detected. Only limited GFAP was stained, localized to the inner limiting membranes and nerve fiber layers at different observed time points in all injected eyes, as in normal retinas. Increased GFAP expressed by activated glial cells in retinas is a nonspecific marker of neuronal injury, ${ }^{20}$ which has been proposed as an important factor contributing to neuron death. ${ }^{25}$ The results in this study revealed that the microspheres and the degraded products did not trigger glial cell activation or further cell death in the retinas. The results also demonstrated the safety of released protein drug EPO in the vitreous.

The retinal microstructure and ultrastructure changes possibly induced by the microspheres and the degraded products were evaluated by retinal HE staining and TEM. The results showed that the retinas remained intact in groups of different doses of microspheres and EPO-dextran PLGA/PLA microspheres with no inflammatory cell invasions. No significant cellular organ changes, such as mitochondria swelling or rupture in photoreceptor inner segments, disc swelling, or disorganization in photoreceptor outer segments, or pyknosis or karyolysis in retinal ganglion cells, were found in TEM analysis. The results indicated that no inflammation or cell toxicity in the retinas was triggered by the microspheres, the degraded products, or the changed microenvironments in the eyes. The ocular tissues showed great tolerability and biocompatibility after the intravitreal administration. These results might be due to the special characteristic of immune privilege in the eyes, which display unique features of blood-ocular barrier anterior chamber-associated immune deviation. This deviation refers to a phenomenon in which antigenic material introduced into the anterior chamber of the eye elicits a systemic immune response that results in immune deviation, characterized by the suppression of T-cell-mediated immunity, while enabling the production of noncomplement-fixing antibodies. ${ }^{26-28}$ Further, this immune response results in reduced expression of major histocompatibility complex molecules on ocular cells, the existence of an intraocular anti-inflammatory environment mediated by resident cells, and various surface-bound and soluble molecules; these serve to modulate the activity of infiltrating immune cells in situ. ${ }^{27,29,30}$

To investigate retinal function changes influenced by the different doses of microspheres and the degraded products, binocular ERG examination was evaluated at 1, 2, 4, 8, and 12 weeks after monocular intravitreal injection. Note that ERG A-wave is obtained primarily from the maximal combined response and reflects the photoreceptor function. Physiologically, A-wave arises from the light-evoked 
closure of sodium channels along the outer segment plasma membrane of photoreceptor cells. The B-wave results from the current flow along Müller cells in response to increased extracellular potassium ion concentrations. It is highly dependent on bipolar cells within the inner nuclear layer and hence on the retinal circulation. ${ }^{31}$ In this present study, the scotopic and photopic ERG amplitudes of neither A- nor $\mathrm{B}$-waves were affected by the microspheres. These findings confirmed that intravitreal injection of PLGA/PLA microspheres had nontoxic effects on retinal neurons and was biocompatible and safe enough for delivering drugs to the posterior segments of eyes.

\section{Conclusion}

This study demonstrated that intravitreal injection of a PLGA/PLA microspheres drug delivery system is biocompatible and safe in normal New Zealand rabbits. The microspheres and the degraded products did not cause cell death, glial activation, or inflammatory reactions in retinas and did not trigger any retinal histological changes or cell ultrastructure damage. Furthermore, these microspheres in the vitreous did not cause retinal function damage. Therefore, the PLGA/PLA microspheres offer a feasible, biocompatible, and safe drug delivery system suitable for intravitreal injection to treat posterior segment ocular diseases. However, to better understand the therapeutic effect of the drug delivery system, further studies are encouraged in order to investigate the polymer degraded process, the exact microenvironment changes, and the drug-controlled release profile in the vitreous.

\section{Acknowledgments}

The study was supported by the project of National Science Foundation of China (81300806), the Program of Eye and ENT Hospital of Fudan University (EENT-2010-09), and the program of Science and Technology Commission of Shanghai Municipality (13401906900).

\section{Disclosure}

The authors report no conflicts of interest in this work.

\section{References}

1. Foulds W, Moseley H, Eadie A, McNaught E. Vitreal, retinal, and pigment epithelial contribution to the posterior blood-ocular barrier. Trans Ophthalmol Soc UK. 1980;100(3):341-342.

2. Ghate D, Edelhauser HF. Ocular drug delivery. Expert Opin Drug Deliv. 2006;3(2):275-287.

3. Cunha-Vaz JG. The blood-ocular barriers: past, present, and future. Doc Ophthalmol. 1997;93(1-2):149-157.

4. Kearns VR, Williams RL. Drug delivery systems for the eye. Expert Rev Med Devices. 2009;6(3):277-290.
5. Tanito M, Li F, Elliott MH, Dittmar M, Anderson RE. Protective effect of TEMPOL derivatives against light-induced retinal damage in rats. Invest Ophth Vis Sci. 2007;48(4):1900-1905.

6. Rong X, Mo X, Ren T, et al. Neuroprotective effect of erythropoietinloaded composite microspheres on retinal ganglion cells in rats. Eur J Pharm Sci. 2011;43(4):334-342.

7. Froger N, Cadetti L, Lorach H, et al. Taurine provides neuroprotection against retinal ganglion cell degeneration. PLOS ONE. 2012; 7(10):e42017.

8. Ghate D, Edelhauser HF. Barriers to glaucoma drug delivery. J Glaucoma. 2008;17(2):147-156.

9. Prasad AG, Schadlu R, Apte RS. Intravitreal pharmacotherapy: applications in retinal disease. Compr Ophthalmol Update. 2007;8(5): 259-269.

10. Wadhwa S, Paliwal R, Paliwal SR, Vyas S. Nanocarriers in ocular drug delivery: an update review. Curr Pharm Design. 2009;15(23): 2724-2750.

11. Yasukawa T, Ogura Y, Kimura H, Sakurai E, Tabata Y. Drug delivery from ocular implants. Expert Opin Drug Deliv. 2006;3(2):261-273.

12. Sinha V, Trehan A. Biodegradable microspheres for protein delivery. $J$ Control Release. 2003;90(3):261-280.

13. Soppimath KS, Aminabhavi TM, Kulkarni AR, Rudzinski WE. Biodegradable polymeric nanoparticles as drug delivery devices. $J$ Control Release. 2001;70(1):1-20.

14. Kreuter J. Nanoparticulate systems in drug delivery and targeting. J Drug Target. 1995;3(3):171-173.

15. Rong X, Yang S, Miao H, et al. Effects of erythropoietin-dextran microparticle-based PLGA/PLA microspheres on RGCs. Invest Ophth Vis Sci. 2012;53(10):6025-6034.

16. Geng Y, Yuan W, Wu F, Chen J, He M, Jin T. Formulating erythropoietin-loaded sustained-release PLGA microspheres without protein aggregation. J Control Release. 2008;130(3):259-265.

17. Andrieu-Soler C, Aubert-Pouëssel A, Doat M, et al. Intravitreous injection of PLGA microspheres encapsulating GDNF promotes the survival of photoreceptors in the rd1/rd1 mouse. Mol Vis. 2005;11: 1002-1011.

18. Shmueli RB, Ohnaka M, Miki A, et al. Long-term suppression of ocular neovascularization by intraocular injection of biodegradable polymeric particles containing a serpin-derived peptide. Biomaterials. 2013;34(30):7544-7551.

19. Jiang C, Moore MJ, Zhang X, Klassen H, Langer R, Young M. Intravitreal injections of GDNF-loaded biodegradable microspheres are neuroprotective in a rat model of glaucoma. Mol Vis. 2007;13: $1783-1792$.

20. O'Callaghan J. Assessment of neurotoxicity: use of glial fibrillary acidic protein as a biomarker. Biomedical and environmental sciences: BES. 1991;4(1-2):197-206.

21. Peng Y, Ang M, Foo S, et al. Biocompatibility and biodegradation studies of subconjunctival implants in rabbit eyes. PLOS ONE. 2011;6(7): e22507.

22. Giarmoukakis A, Labiris G, Sideroudi H, et al. Biodegradable nanoparticles for controlled subconjunctival delivery of latanoprost acid: In vitro and in vivo evaluation. Preliminary results. Exp Eye Res. 2013;112: 29-36.

23. Duvvuri S, Janoria KG, Pal D, Mitra AK. Controlled delivery of ganciclovir to the retina with drug-loaded poly(d,L-lactide-co-glycolide) (PLGA) microspheres dispersed in PLGA-PEG-PLGA gel: a novel intravitreal delivery system for the treatment of cytomegalovirus retinitis. J Ocul Pharmacol Th. 2007;23(3):264-274.

24. Moritera T, Ogura Y, Yoshimura N, et al. Biodegradable microspheres containing adriamycin in the treatment of proliferative vitreoretinopathy. Invest Ophth Vis Sci. 1992;33(11):3125-3130.

25. Woldemussie E, Wijono M, Ruiz G. Müller cell response to laser-induced increase in intraocular pressure in rats. Glia. 2004;47(2):109-119.

26. Kaplan HJ, Streilein JW, Stevens TR. Transplantation immunology of the anterior chamber of the eye II. Immune response to allogeneic cells. J Immunol. 1975;115(3):805-810. 
27. Streilein JW. Ocular immune privilege: therapeutic opportunities from an experiment of nature. Nat Rev Immunol. 2003;3(11):879-889.

28. Niederkorn JY. See no evil, hear no evil, do no evil: the lessons of immune privilege. Nat Immunol. 2006;7(4):354-359.

29. Schewitz-Bowers LP, Lee RW, Dick AD. Immune mechanisms of intraocular inflammation. Expert Rev Ophthalmol. 2010;5(1): $43-58$.
30. Zhou R, Horai R, Silver PB, et al. The living eye "diarms" uncommitted autoreactive $\mathrm{T}$ cells by converting them to Foxp3+ regulatory cells following local antigen recognition. J Immunol. 2012;188(4): 1742-1750.

31. Rosolen SG, Kolomiets B, Varela O, Picaud S. Retinal electrophysiology for toxicology studies: applications and limits of ERG in animals and ex vivo recordings. Exp Toxicol Pathol. 2008;60(1):17-32.

\section{Publish your work in this journal}

The International Journal of Nanomedicine is an international, peerreviewed journal focusing on the application of nanotechnology in diagnostics, therapeutics, and drug delivery systems throughout the biomedical field. This journal is indexed on PubMed Central, MedLine, CAS, SciSearch ${ }^{\circledR}$, Current Contents ${ }^{\circledR} /$ Clinical Medicine,
Journal Citation Reports/Science Edition, EMBase, Scopus and the Elsevier Bibliographic databases. The manuscript management system is completely online and includes a very quick and fair peer-review system, which is all easy to use. Visit http://www.dovepress.com/ testimonials.php to read real quotes from published authors.

Submit your manuscript here: http://www.dovepress.com/international-journal-of-nanomedicine-journal 